PROCEEDINGS OF THE

AMERICAN MATHEMATICAL SOCIETY

Volume 28, Number 2, May 1971

\title{
PARTITION THEOREMS FOR EULER PAIRS
}

\author{
M. V. SUBBARAO
}

ABSTRACt. This paper generalizes and extends the recent results of George Andrews on Euler pairs. If $S_{1}$ and $S_{2}$ are nonempty sets of natural numbers, we define $\left(S_{1}, S_{2}\right)$ to be an Euler pair of order $r$ whenever $q_{r}\left(S_{1} ; n\right)=p\left(S_{2} ; n\right)$ for all natural numbers $n$, where $q_{r}\left(S_{1} ; n\right)$ denotes the number of partitions of $n$ into parts taken from $S_{1}$, no part repeated more than $r-1$ times $(r>1)$, and $p\left(S_{2} ; n\right)$ the number of partitions of $n$ into parts taken from $S_{2}$. Using a method different from Andrews', we characterize all such pairs, and consider various applications as well as an extension to vector partitions.

1. Introduction. Throughout this note, $N$ denotes the set of all natural numbers, $n$ an arbitrary natural number, $r$ an integer $>1$; $S_{i}(i=1,2)$ nonempty subsets of $N ; p\left(S_{i} ; n\right)$ the number of partitions of $n$ into parts taken from $S_{i}$; and $q_{r}\left(S_{i} ; n\right)$ the number of partitions of $n$ into parts taken from $S_{i}$, no part repeated more than $(r-1)$ times at most in any one partition. We write $q_{2}\left(S_{i} ; n\right)=q\left(S_{i} ; n\right)$.

If $q\left(S_{1} ; n\right)=p\left(S_{2} ; n\right)$, George Andrews [1] called $\left(S_{1}, S_{2}\right)$ an Euler pair, after Euler who gave the (probably first) pair (see, for example, $[5$, p. 277]):

$$
S_{1}=N ; \quad S_{2}=\{n \in N \mid n \equiv 1 \quad(\bmod 2)\} .
$$

Two other examples of such pairs:

$$
\begin{aligned}
& S_{1}=\{n \in N \mid n \not \equiv 0 \quad(\bmod 3)\} ; \\
& S_{2}=\{n \in N \mid n \equiv 1,5 \quad(\bmod 6)\}
\end{aligned}
$$

and

$$
\begin{aligned}
& S_{1}=\{n \in N \mid n \equiv 2,4,5 \quad(\bmod 6)\} ; \\
& S_{2}=\{n \in N \mid n \equiv 2,5,11 \quad(\bmod 12)\}
\end{aligned}
$$

are due, respectively to I. J. Schur $[6$, p. 495]) and H. Göllnitz [4, p. 175]. Recently, George Andrews [1, Theorem I], characterized all such pairs by proving the curious (though not unexpected) result:

$$
\begin{aligned}
& \left(S_{1}, S_{2}\right) \text { is an Euler pair if and only if } \\
& 2 S_{1} \subseteq S_{1} \text { and } S_{2}=S_{1}-2 S_{1} .
\end{aligned}
$$

Received by the editors June 25,1970 .

AMS 1969 subject classifications. Primary 1048.

Key words and phrases. Partition, generating function, prime number, quadratic and higher power residues, vector partition.

Copyright (1) 1971, American Mathematical Society 
(As usual, for any $k \in N$, we define $k S_{i}=\left\{k n \mid n \in S_{i}\right\}$ and $S_{1}-S_{2}$ $=\left\{n \in S_{1} \mid n \notin S_{2}\right\}$.)

As Andrews remarked, the uniqueness of the binary representation of natural numbers is equivalent to the statement that, with

$$
S_{1}=\left\{1,2^{n} \mid n \in N\right\} ; \quad S_{2}=S_{1}-2 S_{1}=\{1\},
$$

$\left(S_{1}, S_{2}\right)$ is an Euler pair.

This naturally leads us to consider what analogous interpretation could be given for the uniqueness of representation of natural numbers $n$ to any integral base $r>1$ :

$$
n=\sum_{i=0}^{m} a_{i} r^{i}, \quad a_{i} \in N, \quad 0 \leqq a_{i}<r \quad(i=0,1, \cdots, m), \quad a_{m}>0 .
$$

It is clear that this is equivalent to the property that

$$
q_{r}\left(S_{1} ; n\right)=p\left(S_{2} ; n\right)
$$

with

$$
S_{1}=\left\{1, r^{n} \mid n \in N\right\} ; \quad S_{2}=S_{1}-r S_{1}=\{1\} .
$$

This naturally suggests the following

(1.8) Definition. We say that $\left(S_{1}, S_{2}\right)$ is an Euler pair of order $r$ whenever (1.6) holds.

The purpose of this note is to characterize all such pairs and provide some examples. Towards the end, we briefly consider the extension of the results to vector partitions.

2. A characterization of Euler pairs. Define $q_{r}\left(S_{i}, 0\right)=p\left(S_{i}, 0\right)=1$. Throughout what follows, it is assumed that $|x|<1$. We have

$$
\begin{aligned}
\sum_{n=0}^{\infty} q_{r}\left(S_{1} ; n\right) x^{n} & =\prod_{a \in S_{1}}\left(1+x^{a}+\cdots+x^{(r-1) a}\right) \\
& =\prod_{a \in S_{1}}\left(1-x^{r a}\right)\left(1-x^{a}\right)^{-1} ; \\
\sum_{n=0}^{\infty} p\left(S_{2} ; n\right) x^{n} & =\prod_{b \in S_{2}}\left(1-x^{b}\right)^{-1} .
\end{aligned}
$$

Since the series and products involved are absolutely convergent for $|x|<1$, all the processes in the sequel involving them are valid.

We see at once that $\left(S_{1}, S_{2}\right)$ is an Euler pair of order $r$ if and only if

$$
\prod_{a \in S_{1}}\left(1-x^{r a}\right)\left(1-x^{a}\right)^{-1}=\prod_{b \in S_{2}}\left(1-x^{b}\right)^{-1}
$$


or equivalently,

$$
\prod_{a \in S_{1}}\left(1-x^{r a}\right)=\prod_{a \in S_{1}}\left(1-x^{a}\right) \prod_{b \in S_{2}}\left(1-x^{b}\right)^{-1} .
$$

These relations are useful for obtaining new Euler pairs of various orders. Thus we have the following

(2.5) EXAmple. Let

$$
\begin{aligned}
S_{1} & =\{n \in N \mid n \equiv 1 \quad(\bmod 2)\} \\
S_{2} & =\{n \in N \mid n \equiv \pm 1 \quad(\bmod 6)\}
\end{aligned}
$$

then $\left(S_{1}, S_{2}\right)$ is an Euler pair of order 3 .

While Andrews' main theorem [1, Theorem I] deals only with the case $r=2$, by using arguments similar to his, we obtain the following generalization of his theorem.

(2.6) Theorem. $\left(S_{1}, S_{2}\right)$ is an Euler pair of order $r$ if and only if $r S_{1} \subseteq S_{1}$ and $S_{2}=S_{1}-r S_{1}$.

We shall however prove the theorem making use of the following approach which is not only interesting in itself, but also yields some other results as well. For this reason, we develop it a little beyond our present needs.

Suppose $f(x), g(x)$ and $h(x)$ are (or can be expressed as) power series which are absolutely convergent for $|x|<1$, and that $f(0)$ $=g(0)=h(0)=1$, so that the same holds for their reciprocals. We write $f(x) \equiv g(x)$ to mean that the coefficients of like powers of $x$ on both the sides are equal. Clearly,

$$
f(x) \equiv g(x) \Leftrightarrow f(x) h(x) \equiv g(x) h(x) .
$$

In particular,

$$
f(x) \equiv g(x) \Leftrightarrow(f(x))^{-1} \equiv(g(x))^{-1} .
$$

For later use, we note the following results. Suppose $a_{n}, b_{n}, c_{n}$ $(n=1,2,3, \cdots)$ are increasing sequences of natural numbers, and $s_{n}, t_{n}, u_{n}(n=1,2, \cdots)$ are any sequences of natural numbers. Then

$$
\begin{aligned}
& \prod_{n=1}^{\infty}\left(1-x^{a_{n}}\right)^{-s_{n}} \equiv \prod_{n=1}^{\infty}\left(1-x^{b_{n}}\right)^{-t_{n}} \Leftrightarrow a_{n}=b_{n} ; \quad s_{n}=t_{n} \\
& \quad(n=1,2, \cdots) \\
& \Leftrightarrow \prod_{n=1}^{\infty}\left(1-x^{a_{n}}\right)^{s_{n}} \equiv \prod_{n=1}^{\infty}\left(1-x^{b_{n}}\right)^{t_{n}} .
\end{aligned}
$$




$$
\begin{aligned}
& \prod_{n=1}^{\infty}\left(1-x^{a_{n}}\right)^{-1} \prod_{n=1}^{\infty}\left(1-x^{b_{n}}\right)^{-1} \equiv \prod_{n=1}^{\infty}\left(1-x^{c_{n}}\right)^{-1} \\
& \Leftrightarrow \prod_{n=1}^{\infty}\left(1-x^{a_{n}}\right)^{-1} \equiv \prod_{n=1}^{\infty}\left(1-x^{b_{n}}\right) \prod_{n=1}^{\infty}\left(1-x^{c_{n}}\right)^{-1} .
\end{aligned}
$$

Proof. We shall prove the " $\Rightarrow$ implication" in the first part of (2.9), the reverse implication being trivial. Suppose that $a_{1} \neq b_{1}$. We shall then assume (as we may) that $a_{1}<b_{1}$. Using the relation

$$
\prod_{n=1}^{\infty}\left(1-x^{a_{n}}\right)^{-s_{n}}=\prod_{n=1}^{\infty}\left(1-x^{b_{n}}\right)^{-t_{n}}
$$

and comparing coefficients of $x^{a_{1}}$ on both sides, we obtain the absurd result that $s_{1} a_{1}=0$. Hence $a_{1}=b_{1}$. Again comparing the coefficients of $x^{a_{1}}$ on both sides of (2.11) we get $s_{1}=t_{1}$. We shall now use (2.7) and remove the factor $\left(1-x^{a_{1}}\right)^{-s_{1}}$ from both sides of (2.11) and obtain

$$
\prod_{n=2}^{\infty}\left(1-x^{a_{n}}\right)^{-s_{n}}=\prod_{n=2}^{\infty}\left(1-x^{b_{n}}\right)^{-t_{n}} .
$$

Repeating the above argument, we get successively

$$
a_{n}=b_{n} ; \quad s_{n}=t_{n} \quad(n=2,3, \cdots) .
$$

Note that the second part of (2.9) follows from (2.7). As corollaries to $(2.9)$ we get :

(2.12) If $p\left(S_{1} ; n\right)=p\left(S_{2} ; n\right)$ for all $n \in N$, then $S_{1}=S_{2}$.

ProOF. In the first part of (2.9), take $s_{n}=t_{n}=1$, where $S_{1}$ $=\left\{a_{n} \mid n \in N\right\}, S_{2}=\left\{b_{n} \mid n \in N\right\}$. (The result (2.10) is the lemma in Andrews [1, p. 499].)

To state the next corollary, suppose $E\left(S_{i} ; n\right)$ denotes the excess of the number of partitions of $n$ into an even number of distinct parts over those into an odd number of distinct parts, the parts being taken from the set $S_{i}$. Then

(2.13) If $E\left(S_{1} ; n\right)=E\left(S_{2} ; n\right)$ for all $n \in N$, then $S_{1}=S_{2}$.

This follows from the second part of (2.9) on taking $s_{n}=t_{n}=1$ $(n=1,2, \cdots)$.

Actually, we can generalize (2.9) in many ways. For example,

$$
\begin{aligned}
\prod_{n=1}^{\infty}\left(1-x^{u_{n} a_{n}}\right)\left(1-x^{a_{n}}\right)^{-s_{n}} & \equiv \prod_{n=1}^{\infty}\left(1-x^{u_{n} b_{n}}\right)\left(1-x^{b_{n}}\right)^{-t_{n}} \\
\Leftrightarrow a_{n}=b_{n}, \quad s_{n}=t_{n} & (n=1,2, \cdots) .
\end{aligned}
$$


(As stated previously, $\left(u_{n}\right)$ is any sequence of natural numbers.) The proof of this is similar to the one given before for the first part of (2.10). As a consequence of (2.14), we have, for example, the following result (whose limiting case as $r \rightarrow \infty$ is (2.12)).

(2.15) If $q_{r}\left(S_{1} ; n\right)=q_{r}\left(S_{2} ; n\right)$ for all $n \in N$, then $S_{1}=S_{2}$.

This is obtained from (2.13) on setting $s_{n}=t_{n}=1 ; u_{n}=r(n=1$, $2, \cdots)$.

(2.16) Proof of Theorem (2.6). The "if" part follows because

$$
\begin{aligned}
\prod_{n \in S_{1}}\left(1+x^{n}+x^{2 n}+\cdots+x^{(r-1) n}\right) & =\prod_{n \in S_{1}}\left(1-x^{r n}\right)\left(1-x^{n}\right)^{-1} \\
& =\prod_{n \in S_{1}-r S_{1}}\left(1-x^{n}\right)^{-1} \\
& =\prod_{n \in S_{2}}\left(1-x^{n}\right)^{-1} .
\end{aligned}
$$

The above proof is analogous to that of Andrews [1] for the case $r=2$. But our proof (to follow) for the "only if" part is different from his.

We now assume that $q_{r}\left(S_{1} ; n\right)=p\left(S_{2} ; n\right)$ for all $n \in N$. Suppose that $r S_{1}-S_{1}$ is not empty. We then have

$$
\begin{aligned}
\prod_{n \in S_{2}}\left(1-x^{n}\right)^{-1} & \equiv \prod_{n \in S_{1}}\left(1+x^{n}+\cdots+x^{(r-1) n}\right) \\
& \equiv \prod_{n \in S_{1}}\left(1-x^{r n}\right)\left(1-x^{n}\right)^{-1} \\
& \equiv \prod_{r n \in r S_{1}-S_{2}}\left(1-x^{r n}\right) \prod_{n \in S_{1-r S_{1}}}\left(1-x^{n}\right)^{-1} .
\end{aligned}
$$

Hence, recalling (2.10),

$$
\prod_{n \in S_{2}}\left(1-x^{n}\right)^{-1} \prod_{n \in r S_{1}-S_{1}}\left(1-x^{r n}\right)^{-1} \equiv \prod_{n \in S_{1}-r S_{1}}\left(1-x^{n}\right)^{-1} .
$$

It follows from the first part of (2.9) that every member of $r S_{1}-S_{1}$ is a member of $S_{1}-r S_{1}$; but this is absurd since these two sets are mutually exclusive. Hence $r S_{1}-S_{1}$ is empty, and Theorem (2.6) is proved.

3. Applications. We shall give here some interesting Eulerian pairs as applications of Theorem (2.6).

(3.1) The following pairs are Eulerian of order $r$ :

(3.1.1) $S_{1}=\left\{n \in N \mid n \equiv r, r^{2}(\bmod r(r+1))\right\} ; S_{2}=S_{1}-r S_{1}$.

(3.1.2) $S_{1}=\left\{n \in N \mid n \equiv r, r^{2}, r^{2}+r-1(\bmod r(r+1))\right\} ; S_{2}=S_{1}-r S_{1}$.

For $r=2$, (3.1.2) gives the Göllintz pair described in (1.3). 
(3.2) Let $S_{1} \subseteq N$ be such that $n \in S_{1} \Leftrightarrow r n \in S_{1}$. Let $S_{2}$ $=\left\{n \in S_{1} \mid n \neq 0(\bmod r)\right\}$. Then $\left(S_{1}, S_{2}\right)$ is an Euler pair of order $r$.

The case $r=2$ was previously given by Andrews [1]. In particular, (3.3) $(N,\{n \in N \mid n \neq 0(\bmod r)\})$ is an Euler pair of order $r$.

The pair given in (1.1), originally due to Euler, is a special case of this.

(3.4) Let $r+1$ be a prime. Define

$$
\begin{aligned}
& S_{1}=\{n \in N \mid n \not \equiv 0(\bmod r+1)\}, \\
& S_{2}=\left\{n \in N \mid n \not \equiv r, r+1\left(\bmod r^{2}+r\right)\right\} .
\end{aligned}
$$

Then $\left(S_{1}, S_{2}\right)$ is an Euler pair of order $r$. This includes, in particular, Schur's pair given in (1.2).

As a further application of (3.2) we have

(3.5) THEOREM. Let $p$ be a prime of which $r$ is a quadratic residue. Then the number of partitions of $n$ into quadratic residues $(\bmod p)$, no residue occurring more than $(r-1)$ times at the most in any partition, equals the number of partitions of $n$ into parts which are quadratic residues of $p$ and are not multiples of $r$.

(3.6) Example. The number of partitions of $n$ into parts which are $\equiv 1,3,4,5,9(\bmod 11)$, each part repeated twice at most in any partition, equals the number of partitions of $n$ into parts which are $\equiv 1,4,5,14,16,20,23,25,26,31(\bmod 33)$.

Similar results can be obtained involving cubic and higher power residues.

For example, since 1, 5, 8, 12 are cubic residues modulo 13 we have the following result.

(3.7) $(\{n \in N \mid n \equiv 1,5,8,12(\bmod 13)\},\{n \in N \mid n \equiv 8,12,14,18$, $27,31,34,38(\bmod 39)\})$ is an Euler pair of order 5.

In the results to follow, $x$ and $y$ represent integers.

(3.8) THEOREM. The number of partitions of $n$ into parts which are expressible in the form $x^{2}+2 y^{2}$ equals the number of partitions of $n$ into odd parts which are expressible in the form $x^{2}+2 y^{2}$.

Proof. This follows from Theorem (2.6) in view of the fact that $n$ is expressible in the form $x^{2}+2 y^{2}$ if and only if $2 n$ is [3, p. 68, problem 1].

(3.9) TheOREM. The number of partitions of $n$ into parts which are expressible in the form $x^{2}+x y+y^{2}$, each part repeated at most twice in any partition, equals the number of partitions of $n$ into parts which are relatively prime to 3 and expressible in the form $x^{2}+x y+y^{2}$. 
This follows because $n$ and $3 n$ have the same number of representations in the form $x^{2}+x y+y^{2}[3$, p. 68, problem 2].

4. Extension to vector partitions. The main Theorem (2.6) can be easily extended to vector partitions, that is, partitions of vectors into parts which are vectors. In fact, Euler's original result for the pair given in (1.1) has already been extended in this way by M. S. Cheema [2, Theorem II] as follows:

(4.1) The number of partitions of $\left(n_{1}, n_{2}, \cdots, n_{s}\right)$ into vectors with at least one component odd is equal to the number of partitions of $\left(n_{1}, n_{2}, \cdots, n_{s}\right)$ into distinct parts. The result also holds if the parts are required to have nonzero components.

Our extension of Theorem (2.6) to vectors can be stated thus:

(4.2) The number of partitions of the vector $\left(n_{1}, \cdots, n_{8}\right)$ into parts in which all components belong to $S_{1}$, no part (vector) repeating more than $r-1$ times, equals the number of partitions of the vector $\left(n_{1}, \cdots, n_{s}\right)$ into parts in which all components belong to $S_{2}$ if and only if

$$
r S_{1} \subseteq S_{1} \text { and } S_{2}=S_{1}-r S_{1}
$$

We can further extend the result for the case when the $i$ th component of each part (vector) is required to belong to a set $S_{i}$ which may be different for different values of $i$.

\section{REFERENCES}

1. George E. Andrews, Two theorems of Euler and a general partition theorem, Proc. Amer. Math. Soc. 20 (1969), 499-502. MR 38 \#2112.

2. M. S. Cheema, Vector partitions and combinatorial identities, Math. Comp. 18 (1964), 414-420. MR 29 \#4697.

3. L. E. Dickson, Modern elementary theory of numbers, Univ. of Chicago Press, Chicago, Ill., 1939. MR 1, 65.

4. H. Göllnitz, Partitionen mit Differenzenbedingungen, J. Reine Angew. Math. 225 (1967), 154-190. MR 35 \#2848.

5. G. H. Hardy and E. M. Wright, An introduction to the theory of numbers, 4 th ed., Oxford Univ. Press, London, 1960. MR 20 \#828.

6. I. J. Schur, Zur additiven Zahlentheorie, S. B. Akad. Wiss. Berlin 1926, 488-495.

University of Alberta, Edmonton, Alberta, Canada 